\title{
Aspectos evolutivos da hepatite C pós-transfusional. Revisão de 175 casos
}

\author{
Evolutive aspects of post-transfusion hepatitis C. \\ Revision of 175 cases \\ Henrique Sérgio Mornes Coelho, fátima Aparecida Ferreira figueiredo, Jorge \\ André Segadas, Vera lúcia Panaim, Carmem Martins Nogueira, \\ Cledson Reis Silva e Tationa Jardim Mussi
}

\begin{abstract}
Resumo $O$ vírus da hepatite $C$ é o principal responsável pela hepatite pós-transfusional e sua progressão para hepatite crônica, cirrose e carcinoma hepatocelular é muito comum. A fim de avaliar frequência, tempo e fatores relacionados à progressão da hepatite $C$, estudamos 175 pacientes com hepatite $C$ pós-transfusional. Estes foram divididos em 2 grupos com cirrose $(n=92)$ e sem cirrose $(n=83)$. O tempo médio de desenvolvimento de cirrose foi de $11 \pm 6$ anos. Pacientes com cirrose eram mais velhos à época da transfusão, apresentavam maior prevalência de alcoolismo e tinham tempo de evolução mais longo. O prognóstico foi pior no grupo com cirrose com 28,4\% de mortalidade e 9,1\% de carcinoma hepatocelular, comparados a 5,5\% e 0\% no grupo sem cirrose, respectivamente. Concluímos que a hepatite $C$ pós-transfusional é uma doença progressiva, que se agrava com o passar do tempo, progridindo mais rapidamente em idosos e pacientes com outros fatores de agressão hepática.
\end{abstract}

Palavras-chaves: Hepatite C pós-transfusional. Cirrose. Carcinoma hepatocelular. Epidemiologia.

\begin{abstract}
Hepatitis $C$ virus is the main agent responsable for post-transfusion hepatitis. Progression to chronic hepatitis, cirrhosis and hepatocellular carcinoma is very commom. The aim of this study was to evaluate the frequency, timing and factors related to progression of hepatitis $C$. One hundred seventy five patients with chronic post-transfusion hepatitis $C$ were grouped in a cirrhosis group $(n=92)$ and a non-cirrhosis group $(n=83)$. The medium time of development to cirrhosis was $11 \pm 6$ years. Patients with cirrhosis were older at the time they received transfusion, used more alcohol and had longer times of evolution. The prognosis was worse in the cirrhosis group with a mortality rate of $28.4 \%$ and $9.1 \%$ of evolution towards hepatocellular carcinoma, comparing with $5.5 \%$ and $0 \%$ in the non-cirrhosis group respectively. It is shown that post-tranfusion hepatitis $C$ is slowly developing progressive disease which progress is much more rapidly in elderly patients and patients with others factors of liver damage.
\end{abstract}

Key-words: Post-transfusion hepatitis C. Cirrhosis. Hepatocellular carcinoma. Epidemiology.

Serviços de Clínica Médica e Gastroenterologia do Hospital Universitário Clementino Fraga Filho da Universidade Federal do Rio de Janeiro, Rio de Janeiro, RJ, Brasil.

Endereço para correspondência: Prof. Henrique Sérgio Moraes Coelho. R. Resedá 23/301, Lagoa, 22431-230 Rio de Janeiro, RJ.

Fax: (021) 288-7965.

Recebido para publicação em 07/01/97. 
A hepatite $C$ pós-transfusional é considerada um problema de Saúde Pública no Brasil. Baseados na prevalência do vírus $C$ da hepatite (VHC) de 1,5\% no Brasil12, estimamos que mais de 2 milhões de pessoas estejam infectadas por este vírus. A hepatite pós-transfusional (HPT) foi reconhecida na década de 70 através de estudos prospectivos em pacientes com hepatite associada à transfusão5 9 10. Com a descoberta do VHC, em 1989, logo se determinou que cerca de $90 \%$ das HPT eram causadas pelo $\mathrm{VHC}^{3}$. A infecção aguda pelo VHC não é usualmente detectada, exceto em estudos prospectivos através de dosagens seriadas das transaminases em receptores de transfusões sangüíneas. Elevações mantidas das transaminases por períodos superiores a 6 meses têm sido observadas em cerca de 50 a $80 \%$ dos pacientes com HPT2 14. Estes pacientes são considerados como portadores de hepatite $C$ crônica. Vários estudos sugerem a evolução da infecção aguda pelo VHC para infecção crônica, cirrose e, eventualmente, carcinoma hepatocelular 48 . Entretanto, os resultados são conflitantes quanto à freqüência e ao tempo desta evolução. Koretz e cols 8 em 1983 mostraram evidências de cirrose em $20 \%$ dos pacientes com infecção crônica pelo VHC em 16 anos de seguimento. Por outro lado, Di Bisceglie e cols 4 demonstraram evolução para cirrose em $20 \%$ de 39 pacientes com hepatite crônica num seguimento médio de 9 anos.

Embora hepatite crônica, cirrose e carcinoma hepatocelular sejam seqüelas da infecção pelo VHC, sua freqüência, sua taxa de evolução, os fatores relacionados à taxa de progressão e o grau com que contribuem para a mortalidade destes pacientes não estão totalmente esclarecidos. Para contribuir para o esclarecimento destas questões em nosso contexto, estudamos um grupo de pacientes com infecção crônica pelo VHC referidos a um hospital universitário.

\section{MATERIAL E MÉTODOS}

No período de junho/94 a junho/95, 399 registros de pacientes com testes positivos para anticorpos VHC (Anti-VHC pelo método ELISA 2) e com transaminases elevadas (> $40 \mathrm{U} / \mathrm{L}$ ) foram avaliados pelo Ambulatório de Doenças do Fígado do Hospital Universitário Clementino Fraga Filho. Foram incluídos no estudo somente os 175 pacientes com episódio único de transfusão, a fim de que a data da infecção fosse conhecida. O mês e o ano da transfusão foram obtidos através dos registros médicos. Os 224 pacientes restantes foram excluídos por apresentarem outras fontes de infecção que não permitiram a determinação exata do início da doença como, por exemplo, transfusões em diferentes épocas, uso de drogas endovenosas, hemofilia, hemodiálise. Oitenta e oito pacientes foram positivos para o RNA do VHC pela reação em cadeia da polimerase (método de Garson).

Os pacientes incluídos no estudo foram divididos em 2 grupos: com cirrose (Grupo 1) e sem cirrose (Grupo 2). O diagnóstico de cirrose baseou-se na presença de insuficiência hepática associada à hipertensão portal ou aspecto nodular do fígado na laparoscopia ou presença de nódulos de regeneração na histologia. A ausência destes critérios definiu o grupo sem cirrose, que compreendeu pacientes com hepatite crônica persistente ou hepatite crônica ativa.

Biopsia hepática foi realizada em 117 $(66,8 \%)$ pacientes. Não foi realizada em outros $33(19 \%)$ pacientes, pois os mesmos já preenchiam critérios clínicos de cirrose e/ou testes de coagulação contra-indicavam o procedimento. Em 25 (14,2\%) pacientes não foi realizada por outros motivos como, por exemplo, recusa do paciente.

Com a finalidade de avaliar a progressão da infecção foram estudadas as seguintes variáveis: sexo, idade, idade na época da transfusão, episódio prévio de hepatite aguda ictérica e alcoolismo (uso de 50 gramas de álcool por dia, por pelo menos, 10 anos).

Foram definidos: a) tempo de seguimento: tempo, em meses, da primeira consulta até a última avaliação ou morte; b) tempo de doença: tempo, em anos, da transfusão até a última avaliação ou morte; c) tempo de evolução para cirrose: tempo, em anos, da transfusão até o primeiro diagnóstico de cirrose.

Os resultados são apresentados como média \pm desvio padrão. A análise estatística foi feita pelo teste do Qui-quadrado. Valores de $p<0.05$ foram considerados significativos. $O$ grupo consistiu de $89(51,1 \%)$ pacientes do sexo feminino e $86(48,9 \%)$ do sexo masculino. A média de idade dos pacientes, na última avaliação, foi de $56 \pm 13$ anos. O tempo médio de seguimento foi de 42 meses com uma mediana de 29 meses (1-172). Noventa e dois $(52,6 \%)$ pacientes preencheram os critérios de cirrose e os restantes $83(47,4 \%)$ foram classificados como não cirróticos. A média de 
idade na época da transfusão foi de $43 \pm 14$ anos sendo $46 \pm 12$ anos no grupo 1 e $40 \pm 15$ anos no grupo 2. As características dos 175 pacientes são mostradas na Tabela 1. Todos os pacientes tinham, além da história epidemiológica, pelo menos 6 meses com transaminases elevadas e ausência de outros fatores etiológicos de doença hepática.

\section{RESULTADOS}

O tempo médio de desenvolvimento de cirrose foi de $11 \pm 6$ anos. Ao agruparmos os pacientes em 3 grupos quanto à idade na época da transfusão, encontramos uma diferença estatisticamente significativa quanto ao tempo de progressão para cirrose, sendo $14.1 \pm 7$,

Tabela 1 - Características dos pacientes.

\begin{tabular}{|c|c|c|c|c|}
\hline & $\begin{array}{c}\text { Grupo com cirrose } \\
\qquad \mathrm{n}=92\end{array}$ & $\begin{array}{l}\text { Grupo sem cirrose } \\
\qquad n=83\end{array}$ & $\begin{array}{c}\text { Total } \\
\mathrm{n}=175\end{array}$ & Análise \\
\hline Idade (anos) & $61,8 \pm 11$ & $50,4 \pm 13$ & $56,3 \pm 13$ & $p=0,09$ \\
\hline Sexo $(f / m)$ & $48 / 44$ & $41 / 42$ & $89 / 86$ & $p=0,71$ \\
\hline Idade na época da transfusão (anos) & $46,1 \pm 12$ & $39,6 \pm 15$ & $43 \pm 14$ & $p<0,001$ \\
\hline
\end{tabular}

$10.7 \pm 5,5.6 \pm 3$ anos nos grupos $<40,40-60 \mathrm{e}$ $>60$ anos respectivamente (Tabela 2). Pacientes idosos à época da transfusão apresentaram evolução mais frequente e mais rápida para cirrose (Tabela 3).
A presença ou ausência de alcoolismo foi investigada em 127 pacientes, estando presente em $21 \%(13 / 63)$ no grupo 1 e $11 \%(7 / 64)$ no grupo 2. A análise destes dados nos revela que a freqüência de alcoolismo foi duas vezes maior

Tabela 2 - Relação entre idade na época da transfusão e cirrose.

\begin{tabular}{ccc}
\hline dade (anos) & \% Cirrose & Tempo de evolução para cirrose \\
\hline$<40(24 / 66)$ & 36 & $14,1 \pm 7$ \\
$40-60(55 / 85)$ & 65 & $10,7 \pm 5$ \\
$>60(13 / 24)$ & 54 & $5,6 \pm 3$ \\
\hline
\end{tabular}

$\mathrm{p}<0.001$

\begin{tabular}{|c|c|c|c|c|}
\hline \multirow{3}{*}{$\begin{array}{l}\text { Idade } \\
\text { (anos) }\end{array}$} & \multicolumn{4}{|c|}{ Tempo de evolução } \\
\hline & \multicolumn{2}{|c|}{$<5$ anos } & \multicolumn{2}{|c|}{$<10$ anos } \\
\hline & $\mathrm{n}^{\circ}$ & $\%$ & $\mathrm{n}^{\circ}$ & $\%$ \\
\hline$<40$ & $2 / 24$ & 8,3 & $8 / 24$ & 33,3 \\
\hline$>60$ & $5 / 13$ & 38,4 & $10 / 13$ & 76,9 \\
\hline
\end{tabular}

no grupo com cirrose, embora a comparação entre os grupos não tenha se mostrado estatisticamente significativa $(p=0.13)$.

Cerca de 34\% (53/154) dos pacientes relataram episódio inicial de hepatite aguda ictérica, sendo $30 \%$ no grupo com cirrose $(24 / 79)$ e $39 \%$ no grupo sem cirrose (29/75) (Tabela 4).

O tempo médio de doença pelo $\mathrm{VHC}$ foi de $13 \pm 7$ anos, sendo $14 \pm 6$ anos no grupo $1 \mathrm{e}$ $11 \pm 7$ anos no grupo 2 , variando conforme a idade à época da transfusão. Ao agruparmos os pacientes conforme o tempo de doença pelo
VHC encontramos significativamente maiores porcentagens de cirrose à medida que o tempo progride (Figura 1).

O prognóstico foi muito melhor no grupo sem cirrose onde $94,5 \%$ dos pacientes na última avaliação encontravam-se assintomáticos e apenas 5,5\% morreram, nenhum de causa hepática. No grupo com cirrose, foi possível avaliar a evolução em 88 pacientes, sendo que $28(31,8 \%)$ encontravam-se assintomáticos, apenas com doença histológica e/ou bioquímica ou varizes de esôfago sem sangramento, $35(39,8 \%)$ apresentavam-se com cirrose 


\begin{tabular}{|c|c|c|c|c|}
\hline & \multicolumn{2}{|c|}{ Hepatite ictérica presente } & \multicolumn{2}{|c|}{ Hepatite ictérica ausente } \\
\hline & $\mathrm{n}^{\circ}$ & $\%$ & $\mathrm{n}^{\circ}$ & $\%$ \\
\hline Grupo 1 & $24 / 79$ & 30 & $55 / 79$ & 70 \\
\hline Grupo 2 & $29 / 75$ & 39 & $46 / 75$ & 61 \\
\hline
\end{tabular}

$p=0.27 ; \mathrm{IC}(95 \%)=0,59-1,17$

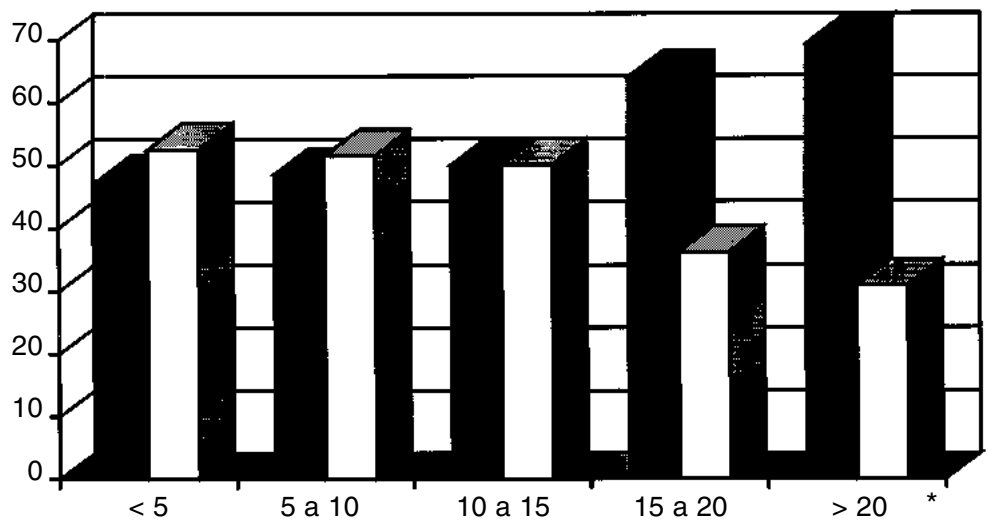

Cirrose

Anos

$\tilde{\mathrm{N}}$ Cirrose

${ }^{*} p=0.03$

Figura 1 - Tempo de doença e cirrose.

descompensada, ou seja, já haviam apresentado, pelo menos, um episódio de descompensação (HDA, ascite, encefalopatia) e $25(28,4 \%)$ haviam morrido das complicações da cirrose e de carcinoma hepatocelular (Tabela 5). Carcinoma hepatocelular desenvolveu-se em $9,1 \%(8 / 88)$ do grupo com cirrose, em um tempo médio de $15 \pm 2$ anos.

Tabela 5 - Evolução clínica da hepatite C pós-transfusional.

\begin{tabular}{|c|c|c|c|c|}
\hline & \multicolumn{2}{|c|}{ Grupo com cirrose } & \multicolumn{2}{|c|}{ Grupo sem cirrose } \\
\hline & $\mathrm{n}^{\circ}$ & $\%$ & $\mathrm{n}^{\circ}$ & $\%$ \\
\hline Assintomático & $28 / 88$ & 31,8 & $67 / 71$ & $94,5^{\star}$ \\
\hline Cirrose clínica & $35 / 88$ & 39,8 & 0/71 & $0^{*}$ \\
\hline Morte & $25 / 88$ & 28,4 & $4 / 71$ & $5,5^{\star}$ \\
\hline
\end{tabular}

${ }^{*} p<0.001$

\section{DISCUSSÃO}

A hepatite pelo vírus $\mathrm{C}$ é a causa predominante de hepatite pós-transfusional, uma doença de importância considerável visto sua alta freqüência e sua tendência à cronicidade, sendo o passo inicial para o desenvolvimento de doença hepática crônica e de alguns casos de carcinoma hepatocelular. Necessitamos estabelecer os fatores preditivos desta evolução, pois a identificação precoce dos pacientes mais prováveis de progredir para cirrose nos permitiria também, precocemente, o início da terapia antiviral.

No Hospital Universitário da UFRJ, de um modo geral, existem 2 fontes de pacientes AntiVHC positivo: os que entram no Hospital pela Triagem do Banco de Sangue (assintomáticos) e os que procuram o Hospital por sinais ou sintomas de doença hepática crônica. Pelo fato da hepatite $C$ ser predominantemente assintomática e de nosso hospital ser um centro de referência, estamos incorrendo em um viés 
de seleção, tendendo a estudar os pacientes mais graves. Isto ficou claro na alta incidência de pacientes com cirrose e no seu curto tempo médio de evolução para cirrose quando comparado a outros estudos 1317.

Em nosso meio, infecção por via póstransfusional é a principal fonte de infecção pelo VHC. Nosso trabalho mostrou que de 399 pacientes com infecção pelo vírus da hepatite $\mathrm{C}$, mais de $50 \%$ eram decorrentes de HPT, ao contrário de países europeus e dos EUA, onde, atualmente predominam as formas esporádicas.

Não houve diferença na progressão da doença em relação ao sexo. Este dado é controverso na literatura com alguns relatos mostrando pior evolução no sexo feminino ${ }^{11} \mathrm{e}$ outros no sexo masculino1. Os pacientes com cirrose possuem uma média de idade maior, talvez evidenciando que há necessidade de um tempo de doença maior para o desenvolvimento de cirrose.

A maioria dos pacientes com HPT C, aguda ou crônica, é assintomática. Em nossa série, apenas $1 / 3$ dos pacientes relataram episódio de hepatite aguda ictérica e isto não mostrou relação com o posterior desenvolvimento de cirrose. $\mathrm{O}$ grupo sem cirrose permaneceu assintomático em sua totalidade até a fase em que desenvolveu cirrose. Neste momento, apresentaram, em geral, sinais de hipertensão porta, encefalopatia hepática, ascite, hiperesplenismo ou coagulopatia. Diversos estudos 4815 demonstraram que embora a maioria dos pacientes com hepatite $C$ crônica seja assintomática, quando é realizada a biopsia hepática, cerca de $50-60 \%$ apresentam hepatite crônica ativa e $15-25 \%$ tem cirrose estabelecida. Contudo, apesar dos achados histopatológicos, há incerteza sobre a progressão à doença clinicamente significante e potencialmente fatal.

Álcool parece ser um co-fator importante para progressão da doença16. Encontramos uma freqüência de $20 \%$ no grupo com cirrose e $10 \%$ no grupo sem cirrose. Portanto, apesar do pequeno número, notamos o dobro de alcoolismo nos pacientes com cirrose corroborando as evidências que ele atue como um fator acessório no desenvolvimento de cirrose.

Pacientes com progressão mais rápida para cirrose tendiam a ser mais velhos na época da transfusão, um achado idêntico a outras séries 1117. Em nossa série, este tempo foi de $14.1 \pm 7,10.7$ \pm 5 e $5.6 \pm 3$ anos para grupos de pacientes abaixo de 40 , entre $40-60$ e acima de 60 anos respectivamente, diferença estatisticamente significativa. A doença parece ser mais grave em indivíduos que adquirem a doença com mais de 60 anos, pois $38,4 \%$ dos pacientes com cirrose nesta faixa etária haviam sido transfundidos há menos de 5 anos, comparados à apenas $8,3 \%$ daqueles com cirrose com menos de 40 anos. Titó e cols 17 , seguindo 303 pacientes com hepatite crônica $\mathrm{C}$ por um período de $95 \pm 43$ meses, constataram evolução para cirrose em 84 (27\%) pacientes, sendo mais freqüente em pacientes com mais de 45 anos. Isto mostra que a idade na época da transfusão pode ser usada como um fator preditivo da evolução para cirrose, provavelmente, associada a uma menor capacidade neutralizadora dos anticorpos. Assim devemos avaliar com cuidado a tendência a não tratar os pacientes idosos, visto que estes são os que mais rapidamente e mais freqüentemente evoluem para cirrose. Por outro lado, isto deve ser pesado contra o fato destes pacientes apresentarem menor resposta a terapia antiviral.

À medida que o tempo de doença progride encontramos uma maior freqüência de cirrose nos pacientes com HPT C, denotando a natureza progressiva, mesmo que indolente, desta patologia. Encontramos cirrose em cerca de $50 \%$ dos pacientes com menos de 15 anos de doença e em quase $70 \%$ dos pacientes com mais de 15 anos de evolução. Jawal e cols6 demonstraram cirrose hepática à biopsia de 18 dentre 30 (60\%) pacientes com hepatite $\mathrm{C}$ com mais de 20 anos de evolução.

Cerca de $9 \%$ dos pacientes do grupo cirrótico desenvolveram carcinoma hepatocelular. Kiyosawa e cols 7 em 1990 registraram que uma proporção significante de pacientes com hepatite crônica $\mathrm{C}$ evoluíram com carcinoma hepatocelular, portanto, esta infecção não tem um curso benigno em muitos pacientes. Nosso tempo médio de desenvolvimento de carcinoma hepatocelular foi de $15 \pm 2$ anos, muito curto comparado, por exemplo, ao relato de Tong e cols 18 que haviam relatado um tempo médio de evolução para carcinoma hepatocelular de 28,3 anos em média.

A mortalidade foi significativamente maior no grupo cirrótico. A freqüência de mortes por causa hepática foi ausente no grupo sem cirrose e prevalente no grupo com cirrose $(28,4 \%)$. O fato do paciente apresentar cirrose implicou em pior prognóstico, embora cerca de 31,8\% destes pacientes fossem assintomáticos (doença 
bioquímica e/ou histológica) à época do diagnóstico.

Os resultados sugerem que idade na época da transfusão, associação com alcoolismo e tempo de hepatite $\mathrm{C}$ influenciam a evolução para cirrose. Sexo, episódio prévio de hepatite aguda ictérica não foram preditores do curso da doença. Pacientes mais velhos na época da transfusão, com história de alcoolismo e maior tempo de evolução de doença foram mais propensos a desenvolver cirrose e em um tempo mais curto. A presença de cirrose implicou em um pior prognóstico com aumento da morbi e mortalidade. Entretanto, outros fatores, além dos relacionados ao hospedeiro, como fatores relacionados ao vírus (tipo de genoma, carga viral, grau de mutabilidade) e ao ambiente poderão ajudar a explicar a evolução da doença hepática pelo VHC.

\section{REFERÊNCIAS BIBLIOGRÁFICAS}

1. Alter HJ. Chronic consequences of non-A, non-B hepatitis. In: Seeff LB, Lewis JH (eds.) Current perspectives in hepatology: Festschrift for Hyman J. Zimmerman, M.D. New York: Plenum Medical p. 83-97, 1989.

2. Berman $M$, Alter HJ, Ishak KG, Purcell $R H$, Jones EA. The chronic sequelae of non- $A$, non-B hepatitis. Annals of Internal Medicine 91:1-6, 1979.

3. Choo QL, Kuo G, Weiner AJ, Overby LR, Bradley DW, Houghton $\mathrm{M}$. Isolation of a cDNA clone derived from a blood-borne non-A, non-B viral hepatitis genome. Science 244:359-362, 1989.

4. Di Bisceglie AM, Goodman ZD, Ishak KG, Hoofnagle $\mathrm{JH}$, Melpolder JJ, Alter HJ. Long-term clinical and histopathological follow-up of chronic posttransfusion hepatitis. Hepatology 14:969-974, 1991.

5. Dienstag JL. Non-A, non-B hepatitis. I. Recognition, epidemiology, and clinical features. Gastroenterology 85:439-462, 1983.

6. Jamal H, Penilo R, Regenstein F. Natural history and morbidity of chronic hepatitis $\mathrm{C}(\mathrm{CHC})$ in a terciary referral practice. Gastroenterology AASLO: A1091, 1995.
7. Kiyosawa K, Sodeyama T, Tanaka E, Gibo Y, Yoshizawa K, Nakano Y, Furuta S, Akahane Y, Nishioka K, Purcell RH, Alter HJ. Interrelationship of blood transfusion, non-A, non-B hepatitis and hepatocellular carcinoma: analysis by detection of antibody to hepatitis $C$ virus. Hepatology 12:671-675, 1990.

8. Koretz RL, Abbey H, Coleman E, Gitnick G. Non-A, non-B post-transfusion hepatitis: looking back in the second decade. Annals of Internal Medicine 119:110115, 1993.

9. Koretz RL, Stone O, Gitnick GL. The long-term course of Non-A, non-B post-transfusion hepatitis. Gastroenterology 79:893-898, 1980.

10. Koretz RL, Stone O, Mousa M, Gitnik GL. Non-A, non-B posttransfusion hepatitis: a decade later. Gastroenterology 88:1251-1254, 1985.

11. Koretz RL, Suffin SC, Gitnick GL. Post-transfusion chronic liver disease. Gastroenterology 71:796-803, 1976.

12. Ministério da Saúde. Sangue - Boletim Epidemiológico 2:31, 1993.

13. Prince AM, Brotman B, Grady GF, Kuhns WJ, Hazzi C, Levine RW, Millian SJ. Long-incubation posttransfusion hepatitis without serological evidence of exposure to hepatitis B virus. Lancet II:241-246, 1974.

14. Rakela JJ, Redeker AG. Chronic Liver Disease after acute non-A, non-B viral hepatitis. Gastroenterology 77: 1200-1202, 1979.

15. Takahashi M, Yamada G, Miyamoto R, Doi T, Endo H, Tsuji T. Natural Course of Chronic Hepatitis C. American Journal of Gastroenteroly 88:240-243, 1993.

16. Tassopoulos NC, Hatzakis A, Delladetsima I, Koutelou MG, Todoulos A, Miriagou V. Role of hepatitis $C$ virus in acute non-A, non-B hepatitis in Greece. Gastroenterology 102:969-972, 1992.

17. Titò L, Sanchez JM, Costa J. L ong-term follow-up of chronic NANB (C) Hepatitis. Journal of Hepatology 11:561, 1990.

18. Tong MJ, El-Farra NS, Reikes AR, Co RL. Clinical outcomes after transfusion-associated hepatitis C. 